Check for updates

Cite this: RSC Adv., 2017, 7, 22900

\title{
Remarkably improved electromechanical actuation of polyurethane enabled by blending with silicone rubber
}

\author{
Christophe Renard, ${ }^{a}$ Dongrui Wang, (D) *a Peng Han, (D) a Silai Xiong, ${ }^{a}$ \\ Yongqiang Wen (D) a and Zhi-Min Dang (D)*b
}

\begin{abstract}
Herein we report the highly improved electromechanical actuation of thermoplastic polyurethane (TPU) by blending with polydimethylsiloxane (PDMS) to construct a bicontinuous structure. TPU/PDMS blend films with various PDMS loadings were fabricated through a simple solution-assisted casting method. Infrared spectroscopy measurements confirmed that TPU and PDMS are thermodynamically incompatible with each other. For $\mathrm{TPU}_{80}$ with 80 parts of PDMS, a bicontinuous phase structure was achieved. The $\mathrm{TPU}_{80}$ film showed greatly decreased elastic modulus and improved elongation at break compared to pristine TPU. It also showed the highest dielectric constant among the TPU/PDMS blend films with various contents of PDMS due to strong interfacial polarization. Most importantly, the $\mathrm{TPU}_{80}$ film exhibited

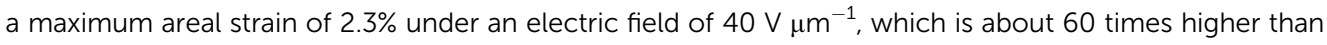
that of pristine TPU. The results described in this work demonstrate that the construction of a bicontinuous interface structure at the micrometer scale is very effective to develop elastomers with superior electromechanical actuation performance.
\end{abstract}

Received 20th March 2017

Accepted 18th April 2017

DOI: $10.1039 / \mathrm{c} 7 \mathrm{ra03274a}$

rsc.li/rsc-advances strong polar molecules ${ }^{15-17}$ have been chemically/physically incorporated into the elastomers. Though the actuation of DEAs can be improved in some extent, the increase in elastic modulus and severe deterioration in dielectric strength limit the practical applications of such elastomers. Over the past few years, it is gradually realized that developing elastomers with low modulus is a more efficient approach to improve the electromechanical actuation. For instance, it is demonstrated that the actuated areal strain of DEAs can be largely enhanced by blending oil-type plasticizers into the rubber matrix. ${ }^{\mathbf{1 8 - 2 0}}$ Very recently, it is reported that silicone rubbers with a bottlebrushlike molecular architecture exhibited ultralow elastic modulus and an over $300 \%$ areal strain under an electric field lower than $10 \mathrm{kV} \mathrm{mm}{ }^{-1} \cdot{ }^{21}$ All these findings confirmed that decreasing the elastic modulus of elastomers to reduce the blocking force is very promising for advanced DEAs.

Among various elastomer candidates for DEAs, polyurethanes (PUs) are gaining increasing interest due to their relatively high permittivity, great breakdown strength, easy processability, and low cost. ${ }^{\mathbf{8 , 1 0 , 2 2}}$ In addition, the versatility of PU chemistry allows tailoring the elastomer's dielectric and mechanical properties in a wide range, which might supply more suitable elastomers for DEAs. ${ }^{23-25}$ The main limitation of PUs as an ideal elastomer is the relatively huge elastic modulus, which is induced by the internal high-density hydrogen bonds. To improve the electromechanical actuation, carbon nanospheres, ${ }^{26}$ carbon nanotubes, ${ }^{27,28}$ graphene nanosheets, ${ }^{28,29}$ and organic strong polar azobenzenes ${ }^{30}$ have been blended into PU 
to construct composite elastomers. However, only limited actuated strain were observed in such PU composite systems due to their further increased elastic modulus and poor dielectric breakdown strength. Recently, Ning et al. have employed an organic molecule, diaminonaphthalene, to blend with PU and disturb the formation of hydrogen bonds. The modified PU with reduced elastic modulus exhibited a max areal strain of $2.6 \% .^{31}$ Very recently, a novel type of PUpolydimethylsiloxane (PDMS) interpenetrating network (IPN) was prepared by chemically grafting PDMS chains with PU chains. ${ }^{32}$ It is interesting to find that elastomers with reduced elastic modulus and enhanced electromechanical strain can be obtained by changing the chain length of PDMS. The optimized PU-based IPN showed a max actuated areal strain of $7.1 \% .^{32}$ However, the complicate chemical synthesis compromises the possibility of such IPN for practical DEAs.

Herein, we investigated the electromechanical actuation of immiscible PU/PDMS blend films prepared by a simple solution casting method with a wide range of composition to tune the micro-structure. A thermoplastic polyurethane elastomer (TPU) combining the low cost, easy processing of thermoplastic materials and elasticity of elastomers has been chosen for the PU. We found a bicontinuous structure of the PU/PDMS blends resulted in remarkably improved actuation strain. The morphology, mechanical properties, and dielectric properties of as-prepared PU/PDMS blends were studied and discussed in detail. We attribute the remarkably improvement in electromechanical actuation to the larger interfacial area in the bicontinuous structure, which is conducive to increase the interfacial polarization.

\section{Experimental section}

\subsection{Materials}

A polyether-based thermoplastic polyurethane elastomer (Elastollan $1185 \mathrm{~A} 囚$ ) (density, $d=1.12$ ) was purchased from BASF and used as received. Tetrahydrofuran (THF), toluene, $\alpha, \omega-$ dihydroxy-terminated PDMS $\left(M_{\mathrm{n}}=90000 \mathrm{~g} \mathrm{~mol}^{-1}, d=0.97\right)$, tetraethylsiloxane (TEOS), and dibutyltin dilaurate (DBTDL) were analytical grade products and used as received.

\subsection{Fabrication of elastomer films}

Plasticized TPU films were fabricated through a solution casting approach as represented in Scheme 1. TPU granules were firstly dissolved in THF and stirred at room temperature to form a homogeneous solution (10 wt\%). Then a certain amount of PDMS was added into the solution and stirred for another $2 \mathrm{~h}$ before the introduction of TEOS as the cross-linker at a concentration of $10 \mathrm{wt} \%$ relative to the PDMS. After incorporation of TEOS, the stirring speed was increased to counteract an increasing in viscosity. After $1 \mathrm{~h}$, the DBTDL catalyst was further added into the solution and stirred for 10 minutes. The resultant solution was casted onto a pre-cleaned glass plate, degassed under vacuum at room temperature for $2 \mathrm{~h}$, and heated at $70{ }^{\circ} \mathrm{C}$ for $24 \mathrm{~h}$. Plasticized TPU films with 20, 40, 60, 80,100 , and 200 parts per hundred resin (phr) of PDMS relative
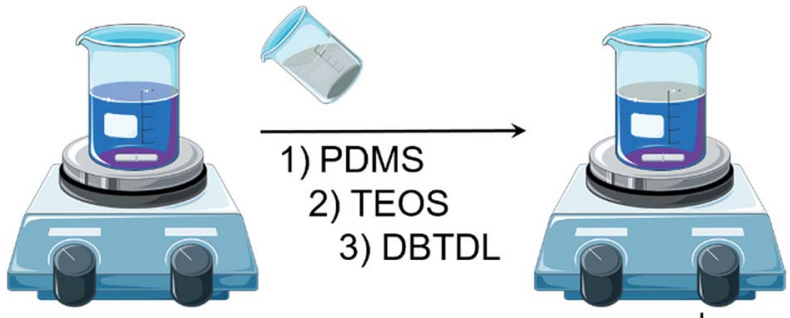

1) Degassed
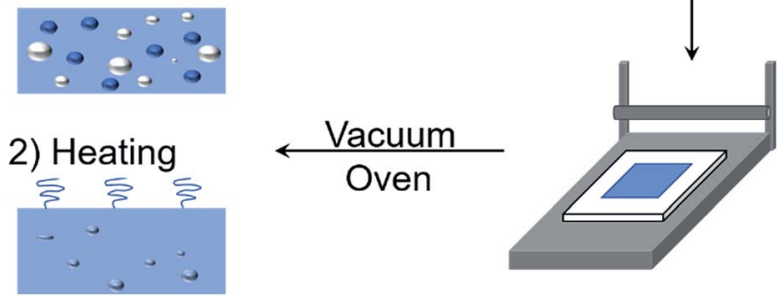

Scheme 1 Fabrication procedures for the PU/PDMS blend films.

to the mass of TPU were prepared. Neat TPU and PDMS films were also prepared as control. As an example, the film made of $1 \mathrm{~g}$ TPU and $0.6 \mathrm{~g}$ PDMS corresponds to $60 \mathrm{phr}$ of PDMS. The films are denoted as $\mathrm{TPU}_{x}$ throughout the manuscript, where $x$ is the content of PDMS (in phr) incorporated into the TPU. For example, the film containing $80 \mathrm{phr}$ of PDMS is denoted as $\mathrm{TPU}_{80}$. The thickness of the films was controlled to be $c a .40 \mu \mathrm{m}$. Noting that the thicknesses of pure PDMS films and $\mathrm{PU}_{200}$ films are slightly higher $(c a .60 \mu \mathrm{m})$ due to the strong adhesion between the films and the glass plate.

\subsection{Characterizations}

The cryo-fractured surfaces of plasticized TPU films were observed by scanning electron microscopy (SEM) using a Hitachi SU8010 under an accelerating voltage of $8 \mathrm{kV}$. Gold was sputtered onto the surfaces of samples prior to tests. Attenuated total reflectance Fourier transform infrared spectra (ATR-FTIR) of samples were measured using a Nicolet 6700 spectrometer equipped with a diamond crystal in the wavenumber range of 600-4000 $\mathrm{cm}^{-1}$. Dielectric constant and loss tangent of the plasticized PU films were measured by using an impedance analyzer (Agilent 4294A) over the frequency range from $1 \mathrm{kHz}$ to $10 \mathrm{MHz}$ under room temperature. Silver paste diluted in xylene were coated as electrodes with an area of $0.8 \times 0.8 \mathrm{~cm}^{2}$ onto the surfaces of the specimens prior to tests. Tensile measurements were performed on an Instron 3365 tensile system under

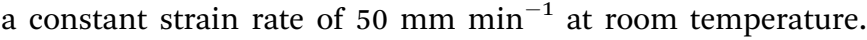
Samples were prepared by cutting the films into a $40 \times 5 \mathrm{~mm}^{2}$ rectangular shape. The Young's modulus $(Y)$ was determined by the slope of stress-strain curves at the strain of $5 \%$.

The electromechanical actuation of the films was evaluated by integrating them into $4 \mathrm{~mm}$ diameter diaphragm-type actuators without pre-strain as described in our previous work. ${ }^{\mathbf{3 0}}$ Graphite powders were coated onto both surfaces of elastomer films as the compliant electrodes. Direct current (DC) high 
voltage was supplied by connecting the actuators to a Boher $\mathrm{HV}$ supplier under a ramping rate of $150 \mathrm{~V} \mathrm{~s}^{-1}$. The voltage signal was monitored and recorded by using an oscilloscope (Tektronix DPO2012B). The actuated out-of-plane displacement was monitored by using a $635 \mathrm{~nm}$ laser position sensor (Panasonic HL-G105) that was focused on the centre of the actuators.

\section{Results and discussion}

\subsection{Morphological structure of the blend films}

The morphology of the TPU/PDMS blends with various PDMS contents was carefully characterized by SEM observation. The blend films were cryofractured and the cross-sections were observed. Typical SEM images are shown in Fig. 1. As expected, typical phase separation morphologies were clearly observed for the blends of the two incompatible polymers. Some wrinkles on the fractured surface are clearly observed for pristine TPU, suggesting the brittle nature of this sample in liquid nitrogen (Fig. 1a). By adding small amounts of PDMS into the TPU matrix, some dispersed spheres with the diameter of several
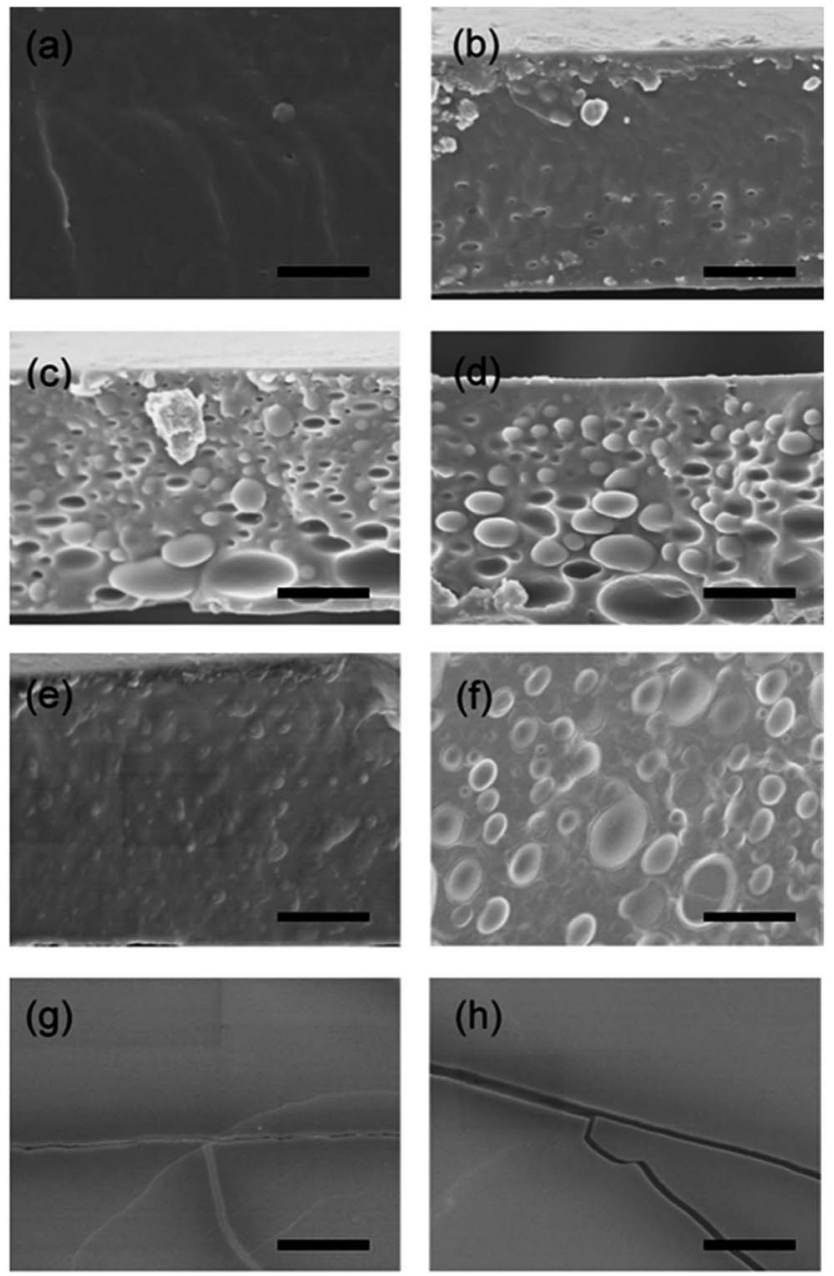

Fig. 1 Typical SEM images of cryofractured cross-sections of (a) TPU, (b) $\mathrm{TPU}_{20}$, (c) $\mathrm{TPU}_{40}$, (d) $\mathrm{TPU}_{60}$, (e) $\mathrm{TPU}_{80}$, (f) $\mathrm{TPU}_{100}$, (g) $\mathrm{TPU}_{200}$, and (h) PDMS. The scale bars correspond to $10 \mu \mathrm{m}$. micrometers are formed throughout the continuous TPU phase (Fig. 1b-d). The size of the spherical phase gradually increases as the increasing of PDMS content. It is also observed that the size of spheres exhibits a gradient distribution in the thickness direction. Conventionally, larger spheres were formed in the bottom side close to the glass substrate, which should be ascribed to the solvent evaporation process. As shown in Fig. 1e, the TPU/PDMS blend with 80 phr of PDMS exhibits relatively smooth cross-section when compared to other samples. The dispersed phase seems to show spherical shape with a smaller averaged diameter than that of $\mathrm{TPU}_{60}$. Considering the volume of PDMS in $\mathrm{TPU}_{80}$ is higher than that of $\mathrm{TPU}_{60}$, this observation suggests that a continuous phase structure of PDMS was formed in $\mathrm{TPU}_{80}$.

To confirm the bicontinuous structure of $\mathrm{TPU}_{80}$, the crosssection was etched by toluene, which is a good solvent for PDMS while a bad solvent for TPU, and further checked. Typical SEM images of TPU blends after toluene etching are shown in Fig. 2. For TPU blends with PDMS content of 20, 40, and $60 \mathrm{phr}$, the morphologies after toluene etching confirm that PDMS is dispersed in TPU matrix with a spherical phase. While for the $\mathrm{TPU}_{80}$, many hollow tubes with diameter of several micrometers are clearly observed after selective extraction of PDMS (Fig. 2d), revealing the PDMS self-organized into a continuous phase throughout the TPU matrix.

To evaluate the morphology evolution of the blends as a function of the volumetric inclusion of PDMS, the content of PDMS in phr was converted into volume fraction. As shown in Fig. 3, $\mathrm{TPU}_{80}$ corresponds to a PDMS volume content of $48 \%$ which is very close to $50 \%$. This can explain well why the bicontinuous structure is obtained for this sample. When the content of PDMS goes beyond 50\%, the phase inversion occurs. In Fig. 1f, it is clear that TPU spheres of several micrometers are uniformly dispersed in the PDMS matrix. In this sample, the volume fraction of PDMS is 53.6\%. By comparing the morphologies of $\mathrm{TPU}_{60}, \mathrm{TPU}_{80}$, and $\mathrm{TPU}_{100}$, one can learn that
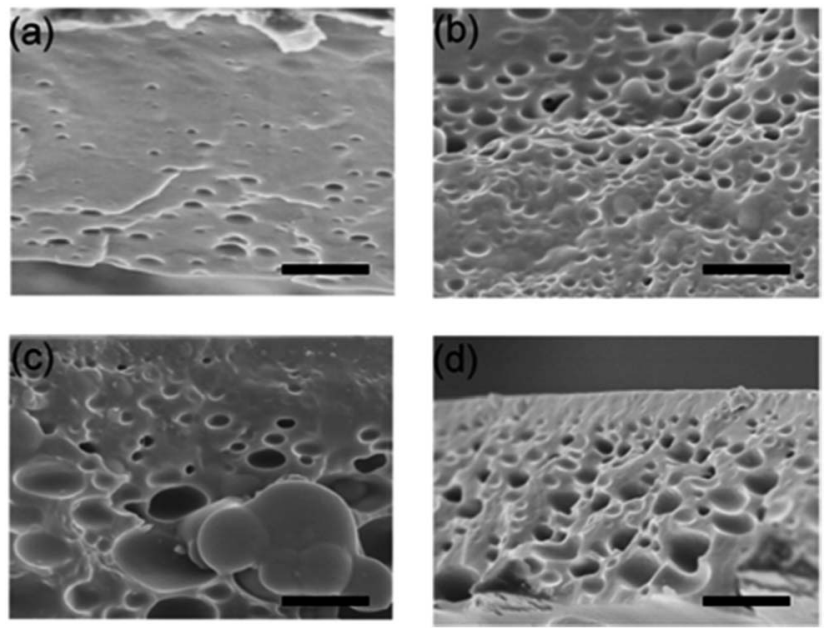

Fig. 2 Typical SEM images of cross-sections of (a) $\operatorname{TPU}_{20}$, (b) $\mathrm{TPU}_{40}$, (c) $\mathrm{TPU}_{60}$, and (d) $\mathrm{TPU}_{80}$ after etched by using toluene. The scale bars correspond to $10 \mu \mathrm{m}$. 


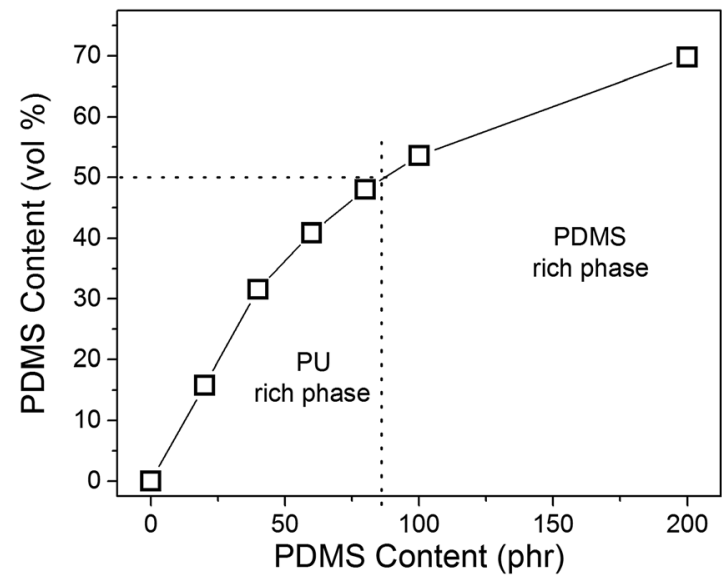

Fig. 3 The relationship between the volume content and the weight content of PDMS in the blends.

the composition range to construct the bicontinuous phase is quite narrow for such TPU/PDMS blends. For $\mathrm{TPU}_{200}$ film, the morphology of its fractured surface is quite similar to that of pure PDMS. Some cracks can be seen on the surface, suggesting the brittle fracture occurred when immersing in liquid nitrogen.

To verify if any interactions exist between TPU and PDMS chains in the blends, infrared spectroscopy was performed on the thin films using an attenuated total reflectance system. The spectra of the TPU blends with different PDMS loadings are shown in Fig. 4. Characteristic peaks corresponding to specific chemical bonds on TPU and PDMS chains are labeled in the figure. Negligible shift in the position of the characteristic peaks was observed, revealing the immiscibility between the two polymers at any given composition. It should be mentioned that the slight shift for the stretching vibration of $\mathrm{C}-\mathrm{O}$ is attributed to the masking effect induced by the intense stretching vibration of $\mathrm{Si}-\mathrm{O}-\mathrm{Si}$ in PDMS, as proved by the ether linkage peak of siloxane in $\mathrm{TPU}_{200}$. However, the specific displacement of the $\mathrm{N}-\mathrm{H}$ stretching vibration to lower wavenumber in $\mathrm{TPU}_{200}$ suggests the breaking of some TPU's hard segment domains into smaller ones induced by the confinement from the PDMS continuous phase. Such poor interactions between the PDMS and the PU have been well demonstrated in the literature dealing with such binary blends. ${ }^{33,34}$

\subsection{Mechanical properties}

Uniaxial tensile tests were performed to evaluate the mechanical behaviour of the blend films, which should be greatly affected by the various morphologies. ${ }^{36}$ Typical stress-strain curves for each sample are summarized in Fig. 5. All the films show the characteristic behaviour of their dominant phase. For blends with PDMS contents lower than $100 \mathrm{phr}$, the introduction of PDMS decreases the Young's modulus and the stress at break of TPU, without a severe reduction in elongation at break. While for the PDMS dominant films, the elongation at break is rather low due to the discontinuity of highly stretchable TPU phase. $\mathrm{TPU}_{200}$ performed poorly as an elastomer which may be caused by the synergetic effect of low content of TPU and the (a)
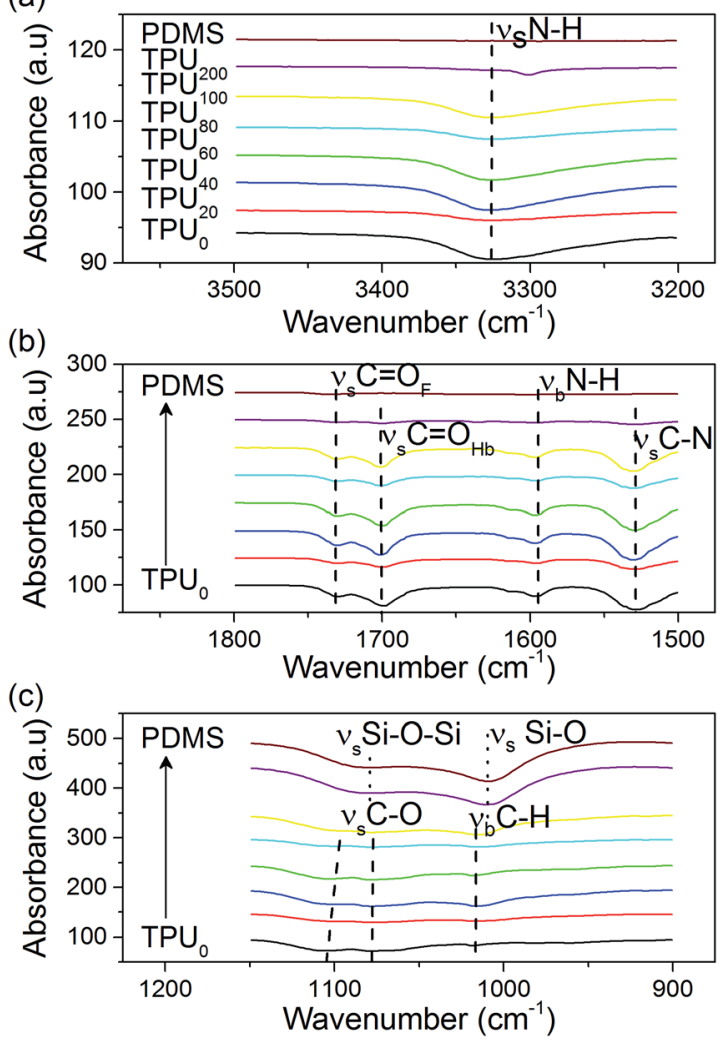

Fig. 4 Infrared spectra of TPU/PDMS blend films in the wavenumber range of (a) $3200-3500 \mathrm{~cm}^{-1}$, (b) $1500-1800 \mathrm{~cm}^{-1}$, and (c) $900-1150$ $\mathrm{cm}^{-1}$. Lines display the positions of different chemical bonds are drawn in the figure to guide the eyes. The subscripts $s$ and $b$ represent stretching and bending, respectively.

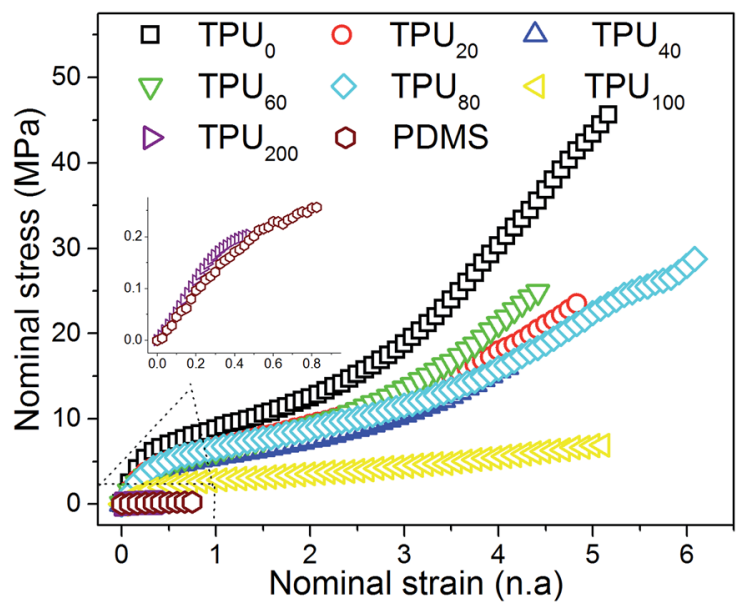

Fig. 5 Stress of TPU/PDMS blend films as a function of strain under uniaxial tensile. The stress-strain curves of pristine TPU and PDMS films are also exhibited for comparison.

decrease of hard segment domain size in it as reflected by the IR spectrum (Fig. 4a). ${ }^{37}$ Surprisingly, the monotonous decreasing trend in the stress and strain at break of the blends by continuous incorporation of PDMS is disrupted for $\mathrm{TPU}_{60}$ and $\mathrm{TPU}_{80}$. It is suspected that the distribution of PDMS inclusions inside 
the TPU for $\mathrm{TPU}_{60}$ could locally form the bicontinuous structure as for $\mathrm{TPU}_{80}$. As compared, the blend $\mathrm{TPU}_{80}$ with the bicontinuous microstructure exhibits the best elongation at break and a moderate Young's modulus. Key data of mechanical properties of the TPU/PDMS blends are listed in Table 1. On the basis of these results, $\mathrm{TPU}_{80}$ seems promising for electromechanical actuation. $^{38,39}$

\subsection{Dielectric properties}

The dielectric properties of the TPU/PDMS blends were tested by using an impedance analyser in the frequency range of $10^{2}$ to $10^{7} \mathrm{~Hz}$ at room temperature. The dielectric constant and loss curves versus frequency are summarized in Fig. 6. In Fig. 6a, strong loss tangent peaks at low frequency side (below $1 \mathrm{kHz}$ ) can be observed for all the blend samples, which should be ascribed to the well-known interfacial polarization. This is also proved by the observation that no strong loss tangent peaks in the spectra of pristine TPU and PDMS. The interfacial polarization comes from the space charges trapped on interfaces of TPU and PDMS phases. ${ }^{22}$ At frequency range of $10^{4}$ to $10^{7} \mathrm{~Hz}$, TPU/PDMS blends show monotonically descending loss tangent as the increase of PDMS content. This should be wellunderstood by the decrease of polar group numbers packing in the blends and the resulting reduction of dipole orientation polarization. All the blends show relatively low loss tangent values $(<0.1)$ in high frequency side, which is acceptable for electromechanical actuation applications., ${ }^{\mathbf{1 , 4}}$

The relative dielectric constant of the blends is shown as Fig. 6b. As expected, the introduction of PDMS with low dielectric constant ( 3.0@1 kHz) decreased TPU's dielectric constant at the measured frequency range. Surprisingly, TPU 200 showed quite low dielectric constant even lower than the pure PDMS. This may be caused by the poor interface between dispersed TPU phase and PDMS matrix, on which some microvoids within trapped air are formed. Except $\mathrm{TPU}_{200}$, other blend samples exhibit reasonable dielectric constant curves. The dielectric constant at $1 \mathrm{kHz}$ of the blends are also listed in Table 1. It can be observed that the $\mathrm{TPU}_{80}$ reached the highest value of 5.1 among the binary blends. Considering the content of PDMS in this sample is even higher than in $\mathrm{TPU}_{20}, \mathrm{TPU}_{40}$,

Table 1 Dielectric loss tangent (tan $\delta$ ) and relative dielectric constant $\left(\varepsilon_{\mathrm{r}}\right)$ at $1 \mathrm{kHz}$, along with the Young's modulus $(Y)$, stress and strain at break $\left(\sigma_{\max }\right.$ and $\left.\lambda_{\max }\right)$ and the calculated electromechanical sensitivity for the different blends

\begin{tabular}{lllllll}
\hline Samples & $\begin{array}{l}\tan \delta @ 1 \\
\mathrm{kHz}\end{array}$ & $\begin{array}{l}\varepsilon_{\mathrm{r}} @ 1 \\
\mathrm{kHz}\end{array}$ & $\begin{array}{l}Y \\
(\mathrm{MPa})\end{array}$ & $\begin{array}{l}\sigma_{\max } \\
(\mathrm{MPa})\end{array}$ & $\begin{array}{l}\lambda_{\max } \\
(\mathrm{n} . \mathrm{a})\end{array}$ & $\begin{array}{l}\beta \\
\left(\mathrm{MPa}^{-1}\right)\end{array}$ \\
\hline $\mathrm{TPU}_{0}$ & 0.05 & 5.8 & 42.0 & 45.9 & 5.20 & 0.14 \\
$\mathrm{TPU}_{20}$ & 0.04 & 3.9 & 14.7 & 24.0 & 4.91 & 0.27 \\
$\mathrm{TPU}_{40}$ & 0.04 & 3.7 & 12.4 & 16.1 & 4.09 & 0.30 \\
$\mathrm{TPU}_{60}$ & 0.05 & 4.6 & 11.5 & 25.0 & 4.47 & 0.40 \\
$\mathrm{TPU}_{80}$ & 0.05 & 5.1 & 8.7 & 29.4 & 6.16 & 0.59 \\
$\mathrm{TPU}_{100}$ & 0.11 & 3.9 & 6.5 & 6.9 & 5.19 & 0.60 \\
TPU$_{200}$ & 0.09 & 2.7 & 2.9 & 0.20 & 0.50 & 0.93 \\
PDMS & $<0.01$ & 3.0 & 0.6 & 0.26 & 0.82 & 5.00
\end{tabular}

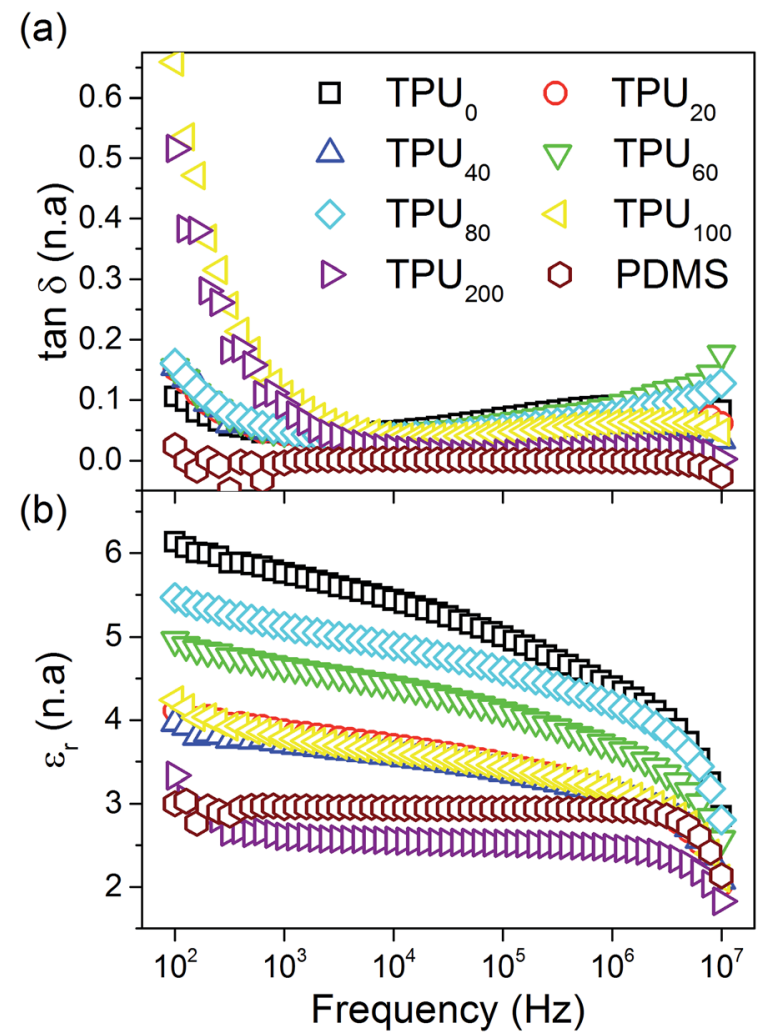

Fig. 6 (a) Dielectric loss tangent and (b) relative dielectric constant of TPU/PDMS blends at room temperature.

and $\mathrm{TPU}_{60}$, the relatively high dielectric constant should mainly benefit from the interfacial polarization.

To clarify the effect of interface on the dielectric constant, we estimated the contact area of PDMS phase and TPU phase in the blends. Using the isoperimetric inequality involving the surface area of an enclose surface with a given perimeter, the product $\Psi \times$ $P$ was taken to reflect the interfacial contact between the inclusion and the volumetric dominant phase. $P$ is the sum of perimeter of all the quasi round inclusions observed in cross-sectional SEM images, which is obtained by calculating the product of their averaged perimeter and number following eqn (1):

$$
P=n \sum_{i=0}^{n} \frac{p_{i}}{n}
$$

where $n$ is the number of quasi round inclusions and $p_{i}$ is the perimeter of the inclusion $i$ and was obtained from the observed diameter of the inclusion $(D)$ in Fig. 1 using:

$$
p=\pi D
$$

While the sphericity $\Psi$, which defines the compactness of a round object, can be calculated following the eqn (3),

$$
\psi=\frac{\pi^{\frac{1}{3}}\left(6 V_{i}\right)^{\frac{2}{3}}}{A_{i}}
$$

where $V_{i}$ and $A_{i}$ represent the volume and surface area of the round inclusion, respectively. For $\mathrm{TPU}_{80}$, considering the continuous structure of the inclusion as an addition of spheres, 
the perfect spherical structure is supposed and the $\Psi$ was assigned to 1 to simplify the calculation. We further assumed that the ellipsoid shapes of dispersed phases in $\mathrm{TPU}_{40}, \mathrm{TPU}_{60}$, and $\mathrm{TPU}_{100}$ led to $\Psi$ values of $0.8,0.9$, and 0.8 , respectively, by considering the deviation from perfect spheres. ${ }^{35}$ To obtain a volumetric representation of the areal interfacial contact, $\Psi \times$ $P$ is further integrated in the whole thickness of the film and divided by its volume and finally denoted as $\Psi \times P_{\mathrm{vol}}\left(\mu \mathrm{m}^{-1}\right)$. The calculated interfacial contact and the $\varepsilon_{\mathrm{r}}$ at $1 \mathrm{kHz}$ for various TPU/PDMS blends are shown in Fig. 7. It is confirmed that the $\varepsilon_{\mathrm{r}}$ is proportional to the interface contact area in the blends, indicating the contribution of interfacial polarization to high $\varepsilon_{\mathrm{r}}$ in such polymer blends is really huge. More importantly, it is found the bicontinuous micro-structure is conducive to improve the interface area and the $\varepsilon_{\mathrm{r}}$ of the blends, which potentially boosts the electromechanical actuation. ${ }^{40}$ By correlation with Fig. 1, the smallest size of PDMS spheres inside the bicontinuous structure leads to the highest interfacial contact by increasing the number of spheres. Considering the research involving the increase in interphase compatibility between TPU and PDMS, ${ }^{41-43}$ one may expect that the introduction of some compatibilizer could either, reduce the content of PDMS to create the bicontinuous phase, or the size of the PDMS spheres. Thus, addition of a compatibilizer could increase the interfacial contact between TPU and PDMS at a given content, inducing higher electromechanical strain comparing to the current system without any compatibilizer.

\subsection{Electromechanical actuation}

To predict the actuation performance of TPU/PDMS blends under a DC electric field, the electromechanical sensitivity $(\beta=$ $\left.\varepsilon_{\mathrm{r}} / Y\right)$ is calculated by using the Young's modulus $(Y)$ and the $\varepsilon_{\mathrm{r}}$ taken at $1 \mathrm{kHz} .{ }^{17,30}$ The results are listed in Table 1 . The obtained data prove that increasing $\beta$ values are obtained as the increasing of PDMS contents in the blends, which suggests that the reduction in $Y$ is more effective for the improvement of electromechanical actuation. The $\mathrm{TPU}_{200}$ and neat PDMS possess the highest $\beta$ values. However, based on the results of

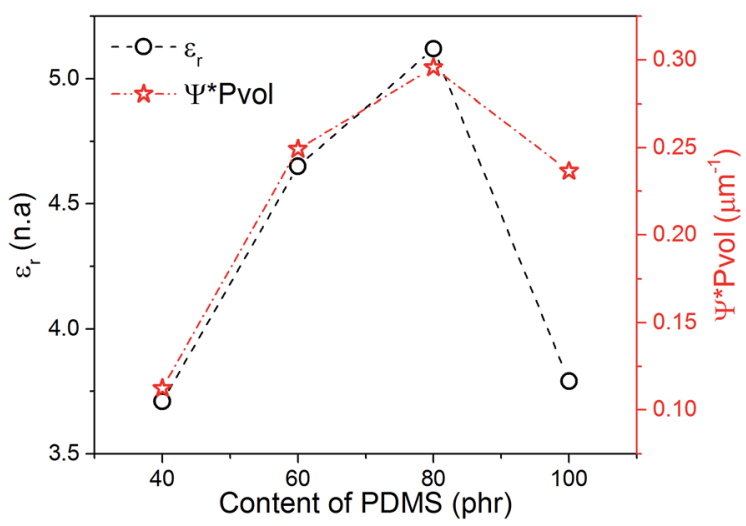

Fig. 7 Variation of the relative dielectric constant and areal interfacial contact between TPU and PDMS $\left(\Psi \times P_{\text {vol }}\right)$ as a function of the PDMS content in the blends. tensile tests, the stretchability of these two samples is rather poor. Therefore, it is doubtful that if high actuated strain can be achieved for these two films.

To verify the validity of the prediction, the experimental actuation of the TPU/PDMS blend films was examined by fabricating them into diaphragm-type actuators as represented in Fig. 8a ${ }^{30}$ Fig. $8 \mathrm{~b}$ displays the experimental displacement of the films as a function of the driving electric field. The experiments were stopped at the electric field strength of $90 \mathrm{~V} \mathrm{\mu m}^{-1}$ if no electrical breakdown occurred before. Except the $\mathrm{TPU}_{200}$ and pure PDMS with poor mechanical properties, all the blend films performed better than pristine TPU. At a given electric field, improved actuated strain can be induced by blending the PDMS and the TPU together even at the lowest content of PDMS (such as $\mathrm{TPU}_{20}$ ). Furthermore, a continuous reduction in required applied electric-field to reach the maximum actuated displacement, following a known trend induced by the reduction in the elastic modulus, can also be seen in Fig. 8b.

In addition, the electromechanical actuation behaviour of the blend films could be classified into two categories depending which polymer acts as the dominant phase. In PDMS-rich blends, the addition of TPU cannot largely increase the actuated displacement. For the $\mathrm{TPU}_{200}$ film, it can be easily understood by taking the seriously deteriorated elasticity into account. However, this is not the case in the TPU 100 . Thus, we suppose that the relatively low actuated strain for the PDMSrich blends is partially caused by the low compatibility

(a)

(b)
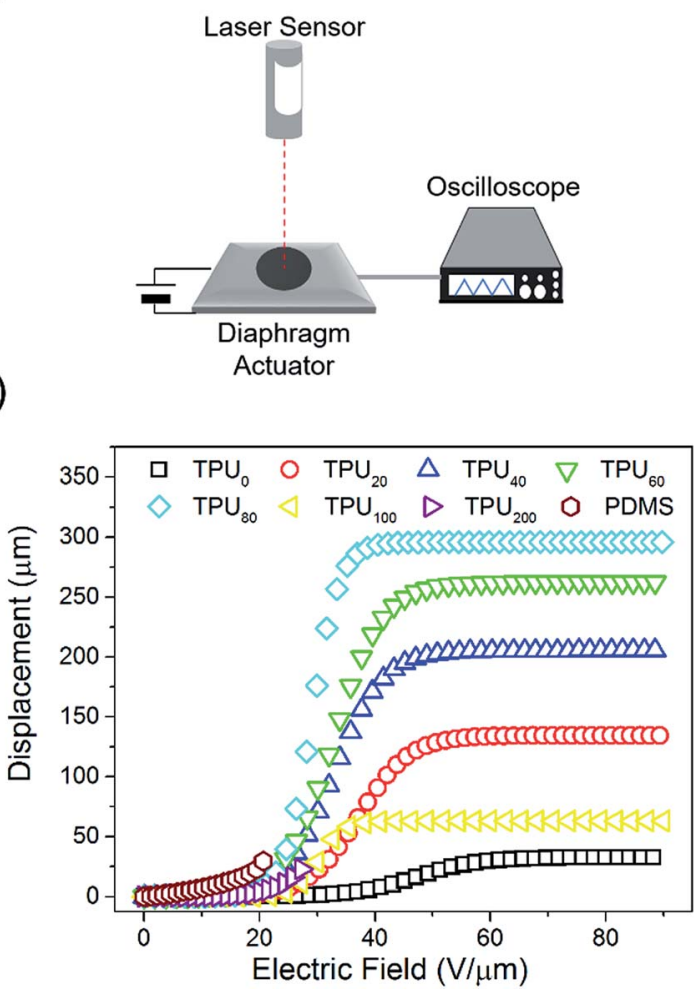

Fig. 8 (a) Schematic representation of a diaphragm-type actuation test, and (b) electric field induced displacement for different TPU/ PDMS blends. 
between the PDMS phase and the compliant electrodes applied onto it. Indeed, even the pure PDMS film behaves worse than those values reported in literature using other type compliant electrode materials..$^{\mathbf{3 9 4 4 , 4 5}}$ However, the study on the compliant electrode was beyond the scope of this work. Moreover, the degree of crosslinking affects the actuation response of PDMSbased DEAs as shown in the literature. ${ }^{\mathbf{4 1 9 , 2 1}}$ Therefore, the current condition of crosslinking should be one reason for such low actuation.

A ten-fold improvement in the maximum displacement is observed for $\mathrm{TPU}_{80}$ when comparing to $\mathrm{TPU}_{0}$, while reducing $30 \%$ of the applied electric field. The highly improved electromechanical actuation is the result of the synergetic effect of dielectric and mechanical properties. Both of them benefit from the bicontinuous structure as discussed previously. Such an improvement may be interesting for designing other type composite elastomers with bicontinuous structures towards advanced DEAs. ${ }^{46}$

The measured actuated displacement was further converted into the areal strain $\left(S_{\mathrm{A}}\right)$ which is defined as follows:

$$
\begin{gathered}
S_{\mathrm{A}}=\lambda_{\mathrm{A}}-1 \\
\lambda_{\mathrm{A}}=\frac{A}{A_{0}}=\frac{\pi\left(R^{2}+d^{2}\right)}{\pi R^{2}}=1+\frac{d^{2}}{R^{2}}
\end{gathered}
$$

where $\lambda_{\mathrm{A}}$ is the ratio between the area at an actuated state $(A)$ and the initial area $\left(A_{0}\right)$ of the films, $R$ is the radius of the diaphragm actuator, and $d$ is the measured displacement. The measured max $S_{\mathrm{A}}$ for the $\mathrm{TPU}_{80}$ film was listed in Table 2 and compared with other similar DEA systems. The TPU/PDMS blend film with a bicontinuous structure developed in this work exhibited a max $S_{\mathrm{A}}$ which is comparable to the literature results. It should be mentioned that for most of DEAs the max $S_{\mathrm{A}}$ values are always achieved under an electric field very close to electrical breakdown. However, TPU/PDMS blends in this work showed the max $S_{\mathrm{A}}$ at an electric field far below the breakdown due to their great insulating properties. As listed in Table 2, the $\mathrm{TPU}_{80}$ film performed better than most of other types of allorganic PU-based elastomers when we compared the $S_{\mathrm{A}}$ at the

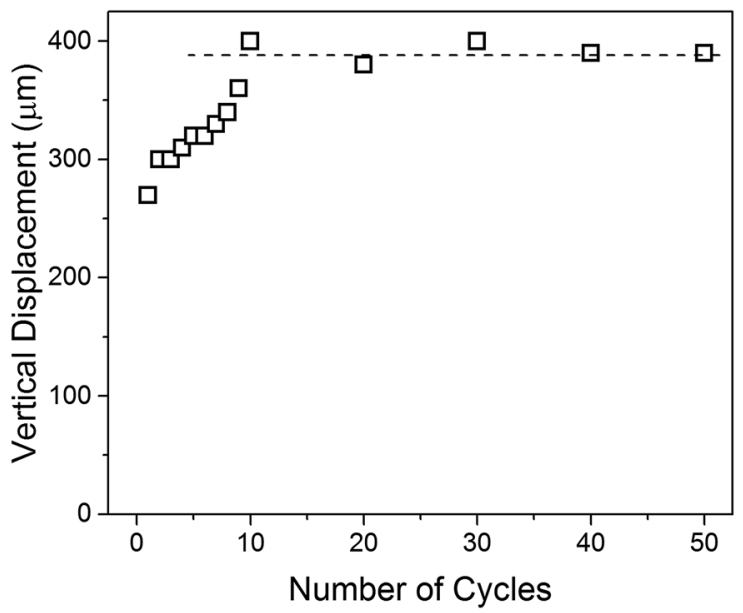

Fig. 9 Continuous electromechanical actuation of $\mathrm{TPU}_{80}$ film under a cyclically linearly changed electric field from $0-35 \mathrm{~V} \mu \mathrm{m}^{-1}$ with a ramp rate of $150 \mathrm{~V} \mathrm{~s}^{-1}$.

electric field equals to $50 \%$ of the electrical breakdown strength $\left(E_{\mathrm{bd}}\right)$.

We also tested the cyclic actuation performance of the $\mathrm{TPU}_{80}$ film. Under a cyclically linearly changed electric field with a ramp rate of $c a .150 \mathrm{~V} \mathrm{~s}^{-1}$, the $\mathrm{TPU}_{80}$ film showed quite stable actuation response after first few activation cycles (as shown in Fig. 9). The stabilized actuated displacement is around $390 \mu \mathrm{m}$ (or $S_{\mathrm{A}}=3.8 \%$ ) at $35 \mathrm{~V} \mu \mathrm{m}^{-1}$, which is slightly higher than the results presented in Fig. 8. Based on the cyclic actuation results, it can be expected that this TPU/PDMS blend with the bicontinuous structure is a good candidate for DEA in long-term practical applications.

\section{Conclusion}

Elastomer films composed of TPU and PDMS were prepared through a simple mixing and solution casting approach. The morphology of as-prepared elastomers can be well tuned by the volume fraction ratio of the two incompatible components.

\begin{tabular}{|c|c|c|c|c|c|}
\hline Elastomers & Actuator form & $\operatorname{Max} S_{\mathrm{A}}(\%)$ & $\begin{array}{l}\text { Electric field@max } \\
S_{\mathrm{A}}\left(\mathrm{V} \mu \mathrm{m}^{-1}\right)\end{array}$ & $\begin{array}{l}S_{\mathrm{A}} @ 50 \% \\
\text { of } E_{\mathrm{bd}}(\%)\end{array}$ & Ref. \\
\hline $\mathrm{TPU}_{0}$ & Diaphragm & 0.04 & 60 & $\ll 0.1$ & This work \\
\hline TPU/disperse red 19 (1 phr) & Diaphragm & $0.8^{a}$ & 57 & $0.1^{a}$ & 30 \\
\hline TPU/diaminonaphthalene (30 phr) & Circular disc & 2.6 & 20 & 1.3 & 31 \\
\hline TPU/poly(ethylene glycol) 600 (80 phr) & Circular disc & 5.2 & 3 & 1.2 & 47 \\
\hline PU/ionic liquid/poly(3,4- & Unimorph & 0.3 & 2 & 0.2 & 50 \\
\hline
\end{tabular}

Table 2 Experimental electromechanical actuation performance of all-organic PU-based DEAs

ethylenedioxythiophene):poly(styrene

sulfonate) (40 wt $\%$ ionic liquid within

$\mathrm{PU})$

${ }^{a}$ Calculated based on the thickness strain using $A / A_{0}=t / t_{0}-1$ under an isochoric condition. 
When the volume ratio of TPU to PDMS approached 1:1, a bicontinuous structure was obtained. The bicontinuous structure remarkably enhanced the interfacial polarization and the dielectric constant of the blend film, that also give rise to a highly improved electromechanical actuation strain. Under the same measurement conditions, the TPU/PDMS blend film with the bicontinuous structure showed a max areal strain of $2.3 \%$, which is about 60 times higher than that of bulk TPU film. More importantly, the highly improved actuation strain can be maintained after 50 cyclic actuation tests. These results demonstrate blend elastomers with micrometer-scale bicontinuous structures are very promising for developing highperformance electromechanical actuators.

\section{Acknowledgements}

Financial support from National Natural Science Foundation of China (21574012, 51425201, 51377010), National Basic Research Program of China (2014CB239505), State Key Laboratory of Power Systems (No. SKLD16Z02), and Fundamental Research Funds for the Central Universities (FRF-BR-16-007A) are gratefully acknowledged.

\section{References}

1 P. Brochu and Q. Pei, Macromol. Rapid Commun., 2010, 31, 10-36.

2 S. Chiba and M. Waki, Recent Advances in Wireless Communications and Networks, InTech, 2011, pp. 435-534, ISBN 978-953-307-274-6.

3 I. A. Anderson, T. A. Gisby, T. G. McKay, B. M. O'Brien and E. P. Calius, J. Appl. Phys., 2012, 112, 041101.

4 J. Biggs, K. Danielmeier, J. Hitzbleck, J. Krause, T. Kridl, S. Nowak, E. Orselli, X. Quan, D. Schapeler, W. Sutherland and J. Wagner, Angew. Chem., Int. Ed., 2013, 52, 9409-9421.

5 A. Khaldi, C. Plesse, F. Vidal and S. K. Smoukov, Adv. Mater., 2015, 16, 4418-4422.

6 C. Larson, P. Beele, S. Li, S. Robinson, M. Totaro, L. Beccai, B. Mazzolai and R. Shepherd, Science, 2016, 351, 1071-1074.

7 R. Pelrine, R. Kornbluh and J. Joseph, Sens. Actuators, A, 1998, 64, 77-85.

8 R. Pelrine, R. Kornbluh, Q. Pei and J. Joseph, Science, 2000, 287, 836-839.

9 C. Keplinger, T. Li, R. Baumgartner, Z. Suo and S. Bauer, Soft Matter, 2012, 12, 285-288.

10 M. Zhenyi, J. I. Scheinbeim, J. W. Lee and B. A. Newman, J. Polym. Sci., Part B: Polym. Phys., 1994, 32, 2721-2731.

11 N. Xu, L. Hu, Q. Zhang, X. Xiao, H. Yang and E. Yu, ACS Appl. Mater. Interfaces, 2015, 7, 27373-27381.

12 M. Poikelispää, A. Shakun, A. Das and J. Vuorinen, J. Appl. Polym. Sci., 2016, 133, 44116-44124.

13 B. Guiffard, D. Guyomar, L. Seveyrat, Y. Chowanek, M. Bechelany, D. Cornu and P. Miele, J. Phys. D: Appl. Phys., 2009, 42, 055503.

14 K. Yang, X. Huang, L. Fang, J. He and P. Jiang, Nanoscale, 2014, 6, 14740-14743.
15 F. B. Madsen, A. E. Daugaard, S. Hvilsted, M. Y. Benslimane and A. Ladegaard-Skov, Smart Mater. Struct., 2013, 22, 104002.

16 M. Dascalu, S. J. Dünki, J.-E. Q. Quinsaat, Y. S. Ko and D. M. Opris, RSC Adv., 2015, 5, 104516-104523.

17 L. Zhang, D. Wang, P. Hu, J.-W. Zha, F. You, S.-T. Li and Z.-M. Dang, J. Mater. Chem. C, 2015, 3, 4883-4889.

18 R. Shankar, A. K. Krishnan, T. K. Gosh and R. J. Spontak, Macromolecules, 2008, 41, 6100-6109.

19 M. Molberg, D. Crespy, P. Rupper, F. Nüesch, J.-A. E. Månson, C. Löwe and D. M. Opris, Adv. Funct. Mater., 2010, 20, 3280-3291.

20 H. Zhao, D.-R. Wang, J.-W. Zha, J. Zhao and Z.-M. Dang, J. Mater. Chem. A, 2013, 1, 3140-3145.

21 M. Vatankhah-Varnoosfaderani, W. F. M. Daniel, A. P. Zhushma, Q. Li, B. J. Morgan, K. Matyjaszewski, D. P. Armstrong, R. J. Spontak, A. V. Dobrynin and S. S. Sheiko, Adv. Mater., 2017, 2, 1604209.

22 Q. M. Zhang, J. Su, C. H. Kim, R. Ting and R. Capps, J. Appl. Phys., 1997, 81, 2770-2776.

23 D. B. Klinedinst, I. Yilgör, E. Yilgör, M. Zhang and G. L. Wilkes, Polymer, 2012, 53, 5358-5366.

24 C. Prisacariu, E. Scortanu and B. Agapie, J. Ind. Eng. Chem., 2013, 19, 113-119.

25 K. Petcharoen and A. Sirivat, Curr. Appl. Phys., 2013, 13, 1119-1127.

26 Y. Yao, N. Ning, L. Zhang, T. Nishi and M. Tian, RSC Adv., 2015, 5, 23719-23726.

27 G. J. H. Melvin, Q. Q. Ni and T. Natsuki, Polym. Compos., 2014, 37, 262-269.

28 T. Chen, L. Pan, M. Lin, B. Wang, L. Liu, Y. Li, J. Qiu and K. Zhu, Polym. Test., 2015, 47, 4-11.

29 S. Liu, M. Tian, B. Yan, Y. Yao, L. Zhang, T. Nishi and N. Ning, Polymer, 2015, 56, 375-384.

30 C. Renard, D. Wang, B.-Z. Han and Z.-M. Dang, RSC Adv., 2015, 5, 82215-82226.

31 N. Ning, B. Yan, S. Liu, Y. Yao, L. Zhang, T. W. Chan, T. Nishi and M. Tian, Smart Mater. Struct., 2015, 24, 032002.

32 C. Tugui, S. Vlad, M. Lacob, C. D. Vargacini, L. Pricop and M. Cazacu, Polym. Chem., 2016, 7, 2709-2719.

33 R. Hernandez, J. Weksler, A. Padsalgikar and J. Runt, Macromolecules, 2007, 40, 5441-5449.

34 M. P. Drupita, K. Naskar and G. B. Nando, RSC Adv., 2016, 6, 80065-80078.

35 J. A. Gopi and G. B. Nando, Advances in Polymer Science and Technology: An International Journal, 2014, 4, 43-51.

36 J. A. Gopi and G. B. Nando, Int. J. Plast. Technol., 2015, 19, 288-308.

37 H. J. Qi and M. C. Boyce, Mech. Mater., 2005, 37, 819-837.

38 H. Stoyanov, M. Kollosche, S. Risse, D. N. McCarthy and G. Kofod, Soft Matter, 2011, 7, 194-202.

39 A. H. A. Razak, P. Szabo and A. Ladegaard-Skov, RSC Adv., 2015, 5, 53054-53062.

40 S. Rudykh, A. Lewinstein, G. Ulner and G. de Botton, Appl. Phys. Lett., 2013, 102, 151905.

41 R. N. Santra, S. Roy, V. K. Tikku and G. B. Nando, Adv. Polym. Technol., 1995, 14, 59-66. 
42 P. Król, B. Król, J. Kozakiewicz, Z. Zapotoczny, B. Pilch-Pitera and S. Kozdra, Prog. Org. Coat., 2015, 81, 72-79.

43 D. Ren, Z. Tu, C. Yu, H. Shi, T. Jiang, Y. Yang, D. Shi, J. Yin, Y.-W. Mai and R. K. Y. Li, Ind. Eng. Chem. Res., 2016, 55, 4515-4525.

44 I. J. Kim, K. Min, H. Park, S. M. Hong, W. N. Kim, S. H. Kang and C. M. Koo, J. Appl. Polym. Sci., 2014, 131, 40030.

45 F. B. Madsen, A. E. Daugaard, S. Hvilsted and A. LadegaardSkov, Macromol. Rapid Commun., 2016, 37, 378-413.
46 K. B. Subramani, E. Cakmak, R. J. Spontak and T. K. Gosh, Adv. Mater., 2014, 26, 2949-2953.

47 M. Tian, B. Yan, Y. Yao, L. Zhang, T. Nishi and N. Ning, J. Mater. Chem. C, 2014, 2, 8388-8397.

48 M. Tian, Y. Yao, S. Liu, D. Yang, L. Zhang, T. Nishi and N. Ning, J. Mater. Chem. A, 2015, 3, 1483-1491.

49 C. Huang, Q. M. Zhang, G. De Botton and K. Bhattacharya, Appl. Phys. Lett., 2004, 84, 4391-4393.

50 H. Okuzaki, S. Tagaki, F. Hishiki and R. Tanigawa, Sens. Actuators, B, 2014, 194, 59-63. 